\title{
Going Through the Rites of Passage: Timing and Transition of Menarche, Childhood Sexual Abuse, and Anxiety Symptoms in Girls
}

\author{
Misaki N. Natsuaki • Leslie D. Leve • \\ Jane Mendle
}

Received: 2 August 2010/Accepted: 7 December 2010/Published online: 24 December 2010

(C) The Author(s) 2010. This article is published with open access at Springerlink.com

\begin{abstract}
Menarche is a discrete, transitional event that holds considerable personal, social, biological, and developmental significance. The present longitudinal study examined both the transition and timing of menarche on the trajectory of anxiety in girls with histories of childhood maltreatment $(N=93 ; 63 \%$ European American, $14 \%$ multiracial, 10\% Latino, 9\% African American, and 4\% Native American). We hypothesized that because menarche is a novel, unfamiliar experience, girls would show greater anxiety around the time of menarche. The anxietyprovoking nature of menarche may be accentuated among earlier-maturing girls and girls with histories of childhood sexual abuse. Results indicated that earlier-maturing girls were more anxious in the pre- and peri-menarche periods than their later-maturing peers; however, their anxiety declined after menarche. Childhood sexual abuse was associated with heightened anxiety throughout this transition. The developmental significance of the timing and transition of menarche in relation to childhood sexual abuse and anxiety is discussed.
\end{abstract}

Keywords Menarche - Puberty - Timing - Transition . Childhood maltreatment $\cdot$ Sexual abuse $\cdot$ Anxiety $\cdot$ Girls

\footnotetext{
M. N. Natsuaki ( $\square)$

Department of Psychology, University of California, Riverside, 900 University Avenue, Riverside, CA 92521, USA

e-mail: misaki.natsuaki@ucr.edu

L. D. Leve

Oregon Social Learning Center, Eugene, OR, USA

J. Mendle

Department of Psychology, University of Oregon,

Eugene, OR, USA
}

\section{Introduction}

Developmental scientists have long been interested in ideas of transitions and timing to explain continuity and discontinuity in psychopathology (Rutter 1996a, b). Transitions are characterized by an event that bridges one stage of life to the next. Timing, on the other hand, mainly concerns a timetable of when a specific transitional event occurs in one's life. These two concepts are intertwined, yet they independently affect the development of psychopathology. Although conceptually distinct, transition and timing are often treated simultaneously in an undifferentiated manner or placed as competing concepts, an oversight that can leave the picture of development incomplete.

The effect of menarche on the emotional adjustment in girls is an exemplar that requires an understanding of the intricate relationship between transition and timing. Menarche is a discrete, transitional event in biosocial development; however, the timing of its occurrence also has developmental implications that influence emotional adjustment. Despite the fact that the concepts of transition and timing are both central to the understanding of the developmental mechanisms linking menarche and psychosocial outcomes, their roles have rarely been examined in a systematically integrated manner. The present study targets both the timing and transition of puberty to elucidate emotional responses during this critical developmental juncture. In particular, we examined trajectories of anxiety during the course of menarcheal transition to specifically address three developmental questions: How does anxiety in girls change before, during, and after menarche? Does anxiety during pre-, peri-, and post-menarcheal phases differ by the timing of this transition? How do associations between anxiety and menarche vary according to early life experiences? Specifically, do girls with histories of 
childhood sexual abuse have a particularly difficult time during this transition?

To examine these questions, anxiety trajectories during the menarcheal transition, in relation to the timing of menarche, were examined in a sample of girls with maltreatment histories. Unraveling the pattern of changes in anxiety during the menarcheal transition is crucial, as the examination of the pattern of change in a developmental system itself constitutes the explanation of the change (Overton 1998). We specifically attempted to disentangle menarcheal timing and transition to explore how normative maturation may be challenging for some children but not for others. In addition, the present study focused on a uniquely high-risk contextual circumstance (i.e., childhood sexual abuse) to elucidate the ways in which high environmental strain may intersect with normal developmental pathways to shape girls' experience of this critical milestone. Results of these analyses hold promise for deepening our awareness of the developmental mechanisms that may help explain the relationships of menarche and anxiety, and for understanding trajectories of risk versus resiliency during this transition.

\section{Menarcheal Transition, Timing, and Anxiety}

Menarche is a developmental milestone that symbolizes the transition from girlhood to womanhood. This transitional event not only involves a biological transformation in girls' bodies that brings qualitative shifts from reproductive immaturity to fertility, but also demands emotional and social adjustments to the new identity and expectations associated with a mature body (Brooks-Gunn and Petersen 1983; Ruble and Brooks-Gunn 1982). Because menarche is an uncertain, ambiguous, and novel event (Caspi and Moffitt 1991), the arrival of a first menstrual cycle is often accompanied by negative feelings (Rembeck et al. 2006), including anxiety, surprise, dismay, panic, confusion (Brooks-Gunn and Ruble 1982; Ruble and Brooks-Gunn 1982), embarrassment, and ambivalence (Moore 1995; Tang et al. 2003). Other morphological changes that occur during pubertal development are continuous and gradual (e.g., breast development, growth spurt, skin changes, growth of pubic hair), but menarche is often sudden, unanticipated, and unplanned. Such an abrupt transition, which is particularly salient in earlier-maturing girls, may provoke anxiety because it does not allow time for emotional preparation (Ruble and Brooks-Gunn 1982). This anxiety, however, seems transient: Although girls may initially report anxiety, ambivalence, and confusion at the peri-menarcheal phase (Ruble and Brooks-Gunn 1982), they eventually adjust (Brooks-Gunn and Ruble 1982). As these studies on the experience of menarche vividly illustrate, menarche can be conceived as a life event that demarcates the boundary between two different stages of life (girlhood to womanhood) and may bring temporary turbulence and some discontinuity in the emotional life of girls. However, these studies - though seminal—are dated; the majority of research on girls' emotional reactions during the menarcheal transition was conducted more than two decades ago (e.g., Ruble and Brooks-Gunn 1982) and children's awareness, responses, and interpretations of menarche may have changed since that point.

While transitional aspects of menarche are crucial to understanding the universal adjustment of girls at puberty, certain individual differences might make the transition to menarche more challenging. First and foremost is the timing of menarche. A substantial body of research has investigated the link between pubertal timing (including age at menarche) and internalizing psychopathology (for reviews, see Ge and Natsuaki 2009; Mendle et al. 2007). With specific regard to anxiety, girls who mature early tend to exhibit more anxiety symptoms and panic attacks (Hayward et al. 1992; Kaltiala-Heino et al. 2003; Reardon et al. 2009). This heightened risk for anxiety among early maturers persists over the course of adolescence and into young adulthood, highlighting the long-term developmental implications of pubertal timing (Graber et al. 2004; Zehr et al. 2007).

One potential explanation of the increased risk for anxiety in earlier-maturing girls is that they are less equipped to handle the challenges and stress of maturation than girls who reach the same developmental milestones at a later chronological age (Caspi and Moffitt 1993; Ge and Natsuaki 2009). Compared to later-maturing counterparts, earlier maturers experience a truncated preparation time to develop resources and skills needed to cope with the array of new stressors that emerge with the acquisition of physical maturity. As a result, earlier-maturing children experience heightened levels of emotional distress. This explanation is readily applicable to the mechanisms that link menarcheal timing and girls' reaction to menarche. Indeed, evidence shows that girls who mature early and/or are unprepared tend to react more negatively to menarche (Ruble and Brooks-Gunn 1982).

There are, however, several questions that have not been addressed in previous work. First, the majority of prior studies conceptualizing menarche as a life event has limited their scope to specific reactions to menarche, excluding how global feelings of anxiety, apprehension, and worry emerge in girls during the months surrounding this transition. Second, prospective, longitudinal designs allowing for a study of process mechanisms by following girls from pre- to post-menarcheal stages are rare (for exceptions, see Brooks-Gunn and Ruble 1982; Ruble and Brooks-Gunn 1982). Given the universality and salience of menarche in girls' lives, how girls navigate the menarcheal 
transition and how the timing of menarche influences this experience warrants revived attention in a longitudinal study that covers the time span before and after menarche. The first goal of the present study was, therefore, to examine the effects of menarcheal timing on trajectories of anxiety during the transition from pre- to post-menarcheal phases.

Sexual Abuse and Anxiety in the Menarcheal Transition

In addition to the timing of menarche, a history of childhood maltreatment, particularly childhood sexual abuse (CSA), contributes to girls' emotional responses to menarche. In general, girls who have experienced CSA tend to manifest a wide array of psychopathologies such as anxiety and depression (e.g., Beitchman et al. 1992; Silverman et al. 1996). In particular, evidence has begun to accumulate, indicating that victims of CSA tend to develop distress and emotional difficulties in the domain of sexual functioning. For instance, victims of CSA report a variety of sexual difficulties later in life, including sexual aversion and ambivalence (Noll et al. 2003), sexual concerns, and sexual adjustment problems (van Bruggen et al. 2006). Given that menarche is a signal that a girl has reached her full reproductive potential, the sexual connotation inherent in menarche may be distressing for girls with histories of sexual trauma. Such negative feelings may develop from unpleasant memories and traumas acquired through CSA (Noll et al. 2003), which may be reactivated in the context of emerging sexuality. Our current knowledge is limited, however, with regard to how CSA influences anxiety in girls during the menarcheal transition. Therefore, the second goal of this investigation was to contribute to the understanding of how anxiety and sexual abuse might be important mechanisms relating to pubertal development across adolescence. This approach to understanding the link between menarche and anxiety by embedding menarche in the context of girls' developmental history (e.g., experiences of CSA in childhood) is in accordance with a growing number of investigators who argue for a more nuanced consideration of the individual social context in which puberty unfolds across development (e.g., Conley and Rudolph 2009; Ge and Natsuaki 2009; Graber 2003; Rudolph and Troop-Gordon 2010).

Although not central to the present study, it is noteworthy that CSA may contribute not only to the nature of the emotional response to menarche, but may also be predictive of the timing of this event. Girls with CSA tend to have menarche earlier than girls who have not experienced CSA (Bergevin et al. 2003; Romans et al. 2003; Turner et al. 1999; Wise et al. 2009; Trickett and Putnam 1993). This is certainly the case for the girls in our sample: As we report in a previous study, girls with CSA reached menarche at an earlier age than girls who experienced other forms of childhood maltreatment but not sexual abuse (Mendle et al. in press). While the precise mechanism by which CSA precipitates an early onset of menarche is not well understood, a number of different hypotheses have been proposed. For instance, according to Belsky et al. (1991), children who are raised in the context of a harsh, stressful rearing environment in childhood have an accelerated reproductive schedule because they develop mistrust toward commitment and investment in social relationships, and eventually adopt a reproductive strategy that permits more conceptions in lifetime. The accelerated reproductive schedule is helpful in their circumstances because it maximizes the reproductive fitness by prolonging the time window for reproductivity and fertility. In contrast to the evolutionary pathway posited by Belsky and colleagues, Trickett and Putnam (1993) suggest that the trauma of CSA may activate the hypothalamicpituitary-adrenal (HPA) and hypothalamic-pituitary-gonadal (HPG) axes prematurely, precipitating an early onset of maturation, and explaining some of the emotional behavioral dysregulation commonly emerge in CSA victims. Although the focus of this study was exclusively on the effects of CSA and menarche on psychopathological outcomes, readers are reminded of the intricate, complex associations among these three concepts.

\section{The Present Study}

The overarching aim of the current study was to examine the effects of menarcheal timing and CSA on trajectories of anxiety during the transitional periods of pre-, peri-, and post-menarche in girls with a history of childhood maltreatment. This study was designed to revisit the earlier literature on girls' reactions to menarche and to expand it by incorporating the role of childhood family environment (i.e., childhood maltreatment) and by paying special attention to both the transition and timing of menarche as critical developmental processes. Several methodological and conceptual strengths were employed. First, the study was based on a uniquely high-risk sample of girls who had experienced highly adverse family environments in childhood. This kind of sample has been largely absent in the puberty literature and its inclusion helps to better elucidate contextual circumstances affecting the experiences of menarcheal transition. Second, the maltreatment incidents were all corroborated by the official documents. Third, this longitudinal study was implemented when many girls in the sample were experiencing menarcheal transition. Therefore, we were able to study the developmental change in anxiety as girls' bodies transformed from pre- to postmenarche. 
Three major hypotheses guided the present study. First, following the literature on early pubertal maturation and psychopathology (e.g., Ge and Natsuaki 2009; Mendle et al. 2007) as well as previous work on girls' reaction to menarche (Ruble and Brooks-Gunn 1982), we hypothesized that earlier-maturing girls would show higher levels of anxiety at the peri-menarcheal phase than their latermaturing counterparts. Second, we expected levels of anxiety to decline gradually in post-menarche. The rationale underlying this hypothesis was that as girls experience and gain knowledge about menarche, the novelty and unknown factors associated with menstruation, which may provoke anxiety, wanes over time. Additionally, we expected the decline in anxiety to be particularly salient for early maturers because their menarcheal status loses its peculiarity over time as an increasing number of later-maturing girls become postmenarcheal. Third, we expected that girls with a history of CSA would be at a heightened risk for anxiety during the transition from pre- to post-menarcheal status, compared to girls with a history of childhood maltreatment of a non-sexual nature (e.g., neglect, physical abuse). We focused on CSA in particular because pubertal development is inherently a part of sexual maturation and the traumatizing prior experience of CSA is a likely developmental risk factor that may lead girls to have anxious reactions to their changing body.

In pursuit of the study goals and the above-mentioned hypotheses, we took three potential covariates into account. First, it is important to acknowledge that in a sample of individuals with maltreatment experiences, such as this present sample, the co-occurrence of multiple maltreatment subtypes is standard (Manly et al. 2001). In order to examine unique effects of CSA, we statistically considered the presence of maltreatment types other than CSA. Second, the effect of chronological age was considered because research has shown that trajectories of anxiety (i.e., generalized anxiety disorder symptoms) follow an upward age trend during adolescence, particularly for girls (Hale et al. 2008). Finally, because this study was a part of an intervention trial in which the participants were randomly assigned to a behavioral support intervention condition or to a regular foster care control condition (Chamberlain et al. 2006), we included the intervention condition as a cluster variable in the analyses. Intervention effects were not a focus of the present study and were not hypothesized to relate to the study variables. We also considered ethnicity as a potential covariate. However, we found no evidence of ethnic differences in age at menarche $[F(4,88)=0.63$, n.s. $]$, anxiety [Time $1, F(4,88)=0.86$, n.s.; Time $2, F(4,88)=0.79$, n.s.; Time $3, F(4$, $83)=0.72$, n.s.], or any other study variables. Therefore, ethnicity was omitted from the primary analyses.

\section{Method}

\section{Participants}

The study included a sample of 93 girls living in statesupported foster homes who were finishing elementary school between 2004 and 2007. All participants had a history of childhood maltreatment and were part of an intervention trial in which they were randomly assigned to a behavioral support intervention condition or to a regular foster care control condition (Chamberlain et al. 2006). The girls were referred through local child welfare systems and were recruited with their foster parents during the spring of their final year of elementary school.

At the inception of the study, the girls were, on average, 11.54 years of age $(\mathrm{SD}=0.48$, range $=10.44-12.92$ ). The ethnic distribution was as follows: $60 \%$ European American, 15\% multiracial, 11\% Latino, $10 \%$ African American, and $4 \%$ Native American. The girls entered foster care at an average age of 7.63 years $(\mathrm{SD}=3.14)$ and spent an average of 2.90 years in foster care $(\mathrm{SD}=2.25)$ at the start of the study. Of the 93 girls in our sample, $80 \%$ had experienced multiple foster care placements, with an average of 3.9 placements $(\mathrm{SD}=3.03$, range $=1.0-18.0$ ).

\section{Procedure}

The present study included three waves of longitudinal data collected at baseline (T1), 12 months (T2), and 24 months (T3). Although the original study consisted of four major assessments (an additional assessment at 6 months after the study began), the present investigation did not include data from the 6-month assessment because information regarding anxiety was not collected at that time point. This study utilized three sources of data, including a standardized interview and questionnaires for each girl, an interview with each girl's caseworker, and the collection of child welfare records. The interviews took approximately $2 \mathrm{~h}$. Retention rates across the study period ranged between 86 and $93 \%$.

\section{Measures}

\section{Anxiety Symptoms}

The Revised Children's Manifest Anxiety Scale (RCMAS; Reynolds and Richmond 1978, 1979) was used to assess the girls' current anxiety symptoms. The RCMAS is a widely-used instrument for assessing the level and nature of anxiety in children and adolescents. It consists of 37 items: nine that measure social desirability in item responses ("Lie" scale) and 28 that measure anxiety in 
factor-analytic subscales (physiological manifestations of anxiety, worry and over-sensitivity, and fear/concentration). Participants circled "yes" for each statement that was true about themselves, and "no" for each statement that was not. The RCMAS has demonstrated high three-week test-retest reliability ( $r=0.90$; Reynolds and Paget 1983) and adequate nine-month test-retest reliability $(r=0.68$; Reynolds 1981). Anxiety items were summed to index the level of anxiety. Internal consistency for the anxiety scale in this study was acceptable $(\alpha=0.87,0.83$, and 0.84 for $\mathrm{T} 1, \mathrm{~T} 2$, and $\mathrm{T} 3$, respectively). In the current sample, 15,8 , and $5 \%$ of the participants manifested a clinical level of anxiety (a $\mathrm{T}$ score of 60 or above) for $\mathrm{T} 1, \mathrm{~T} 2$, and $\mathrm{T} 3$, respectively.

\section{Age at Menarche}

At each wave, girls reported whether they had their first menstrual cycle and, if so, the age (years and months) at which it occurred. By repeatedly inquiring about menarche at all three waves, the study design allowed us to capture the information about girls who had not had their first menstrual cycle at previous time points but had at one of the later time points. Girls who had reached menarche at previous time points were also asked about age at menarche at subsequent time points. The consistency of their menarche reports across the study period was over $95 \%$. We used the first-time reported data, which is the closest to each girl's actual experience of menarche. This strategy helps to reduce telescoping bias (Pickles et al. 1994) in which events are remembered as closer to the date of interview than they actually are.

There were only 25 girls who reached post-menarcheal status by T1. Twenty-nine girls who were pre-menarcheal at $\mathrm{T} 1$ became post-menarcheal by $\mathrm{T} 2$. Eleven additional girls reached post-menarcheal status by T3. A follow-up telephone interview was conducted after the 24-month assessment that inquired about menarche. With 28 additional girls who were pre-menarcheal at T3 becoming postmenarcheal by the follow-up telephone interview, a total of 93 girls reported that they had their menarche by the end of the study. Therefore, $73 \%$ of girls underwent the menarcheal transition during the study period. The mean age of menarche in this sample was 11.99 years $(\mathrm{SD}=1.00)$. In general, the current sample experienced menarche a few months earlier than the recent population of U.S. girls (average age of menarche at 12.4 years; McDowell et al. 2007), which is consistent with research indicating that timing of menarche tends to be accelerated among girls raised in stressful childhood environments (see Belsky et al. 1991; Ellis 2004).

Based on the distribution of age at menarche in the sample, girls were divided into two different menarcheal timing groups (i.e., an earlier- and a later-maturing group). The cut-off was determined by the median split (which was found to be equivalent to the mean) of age at menarche at 12 years (144 months). The median split was used to ensure an adequate sample size for each group. Girls who had their first menstrual cycle before the age of 144 months $(n=44)$ were classified as those who matured earlier than peers. The mean age at menarche of the earliermaturing girls in the current sample was at 133.7 months (11.14 years), which is earlier than the national average in the recent cohort of girls who were born between 1980 and 1984 (the average age at menarche $=12.4$ years, McDowell et al. 2007). Only $10 \%$ of U.S. girls are estimated to have menarche by 11.11 years of age (Chumlea et al. 2003). Girls who had their first menstrual cycle at or after age of 144 months $(n=49)$ were identified as latermaturing. The mean age at menarche in girls who were in the later-maturing group was 153.0 months or 12.8 years old, which was slightly later than the national average (McDowell et al. 2007).

To provide further support for the group classification, we examined the distribution of the Pubertal Development Scale (PDS; Petersen et al. 1988) by different maturation groups. Across three waves, girls completed a slightly abbreviated version of the PDS that comprised three items assessing changes in body hair, skin (e.g., development of pimples and acne), and breast growth. Girls reported their responses using a 4-point Likert scale ( $1=$ no changes yet, $4=$ seems completed). As shown in Table 1 , girls who were classified as earlier maturers scored higher on the PDS items than those who were assigned to the latermaturing group, with two exceptions. Unexpectedly, there was no group difference on the item of body hair growth at $\mathrm{T} 1(p=.24)$ and only a marginal significant difference between groups on the item of skin change at T3 $(p<.10)$. However, in general, girls whose menarche arrived earlier showed more advanced pubertal development in other parts of their bodies (i.e., body hair, skin change, and breast development). These findings provide further validity for the two-group classification by timing of menarche in this sample.

\section{Sexual Abuse}

Childhood maltreatment experiences were based on child welfare records. The official records were coded according to a modified version of the Maltreatment Classification System (MCS; Manly et al. 2001). The MCS system classifies maltreatment cases into subtypes, one of which included sexual abuse. Coders reviewed the child welfare case records to identify incidents of maltreatment. The case had to match the MCS definitions of maltreatment and had to be verified by the child welfare system caseworker in 
Table 1 Means of items in the Pubertal Development Scale by menarcheal timing groups

\begin{tabular}{|c|c|c|c|c|c|c|c|c|c|}
\hline \multirow[t]{2}{*}{ Waves } & \multicolumn{3}{|c|}{ Changes in body hair } & \multicolumn{3}{|c|}{ Skin changes } & \multicolumn{3}{|c|}{ Breast development } \\
\hline & Earlier & Later & $F(d f)$ & Earlier & Later & $F(d f)$ & Earlier & Later & $F(d f)$ \\
\hline $\mathrm{T} 1$ & 3.10 & 2.79 & $(1,87)=1.37$ & 2.59 & 2.10 & $(1,89)=5.85^{* *}$ & 2.55 & 2.17 & $(1,87)=8.32^{* * *}$ \\
\hline $\mathrm{T} 2$ & 3.54 & 2.98 & $(1,85)=4.52^{* *}$ & 2.71 & 2.30 & $(1,86)=5.07^{* *}$ & 2.71 & 2.25 & $(1,86)=12.24 * * *$ \\
\hline T3 & 3.70 & 2.93 & $(1,81)=12.56^{* * *}$ & 2.80 & 2.40 & $(1,83)=3.08^{*}$ & 2.90 & 2.45 & $(1,82)=9.34 * * *$ \\
\hline
\end{tabular}

$* p<.10 ; * * p<.05 ; * * * p<.01$

order to be counted as a maltreatment incident. Two-thirds of the cases were double-coded to check inter-rater reliability, and the inter-rater agreement of sexual abuse was $86 \%$. It is important to note that by the nature of the project, all girls had experienced at least one type of maltreatment before the enrollment in the study.

The sample was unique in that the participating girls experienced unusually high-risk childhood environments. Fifty-three percent of the girls had at least one incident of physical abuse and $77 \%$ had at least one incident of emotional maltreatment. More pertinent to the present study, the current coding scheme revealed that approximately $64 \%$ of the girls $(n=62)$ experienced at least one episode of CSA $(M=2.16$ incidents of CSA per person, $\mathrm{SD}=1.36)$. The remaining one-third of the girls $(n=31)$ reported no history of CSA. We coded the girls with no CSA (but with a history of childhood maltreatment other than CSA) as zero and those with CSA experience as one.

When studying the effects of pubertal timing and childhood maltreatment, researchers must be aware of the confounding nature of CSA and menarcheal timing, especially given that girls who have experienced CSA are more likely to experience menarche earlier than peers. However, under the operationalization of pubertal timing and CSA in the current investigation, we also found that there are variations in age at menarche by CSA status. The results from the cross-tabulation indicated that in girls with a history of CSA, there were 36 earlier maturers and 26 later maturers. In girls without any CSA history, there were 8 earlier maturers and 23 later maturers. Therefore, although girls with a history of CSA generally experienced menarche at an earlier age than girls without a CSA history, there was individual variation.

\section{Gynecological Age}

Because the goal of the study was to examine developmental trajectories of anxiety surrounding menarche, gynecological age was used as a unit of time (a more detailed discussion is provided in a later section). Gynecological age was calculated by subtracting the age at menarche (in months) from the date of the assessments (e.g., Dorn et al. 2009; van Hooff et al. 2000). With three waves of data collection, the study captured the girls' anxiety between 39 months prior to and 46 months after menarche, although the majority of data (83\%) concentrated on the time window of \pm 24 months of menarche.

To increase the number of cases in each time window, we categorized the time variable into eleven phases: five phases of the pre-menarche periods (6 months, 7-12 months, 13-18 months, 19-24 months, and 25+ months prior to menarche), at menarche (peri-menarche, or the intercept), and five phases of the post-menarche periods (6 months, 7-12 months, 13-18 months, 19-24 months, and $25+$ months after menarche). Table 2 presents the number of observations by time since menarche. A total of 274 observations were obtained across the three waves of data collection.

Due to the nature of the study design, it is important to note that earlier-maturing girls may be over-represented in data regarding the post-menarcheal periods (they are more likely to have had menarche before the inception of the study), and later-maturing girls may be over-represented in data regarding the pre-menarcheal periods. To address this possibility, we computed the number of participants by gynecological age and menarcheal timing status (see Table 2). While the distribution of the participants

Table 2 The number of observations over time by menarcheal phase and timing

\begin{tabular}{|c|c|c|c|c|c|c|c|c|c|c|c|c|}
\hline & \multicolumn{5}{|c|}{ Pre-menarche (in months) } & \multirow[t]{2}{*}{ At menarche } & \multicolumn{5}{|c|}{ Post-menarche (in months) } & \multirow[t]{2}{*}{ Total } \\
\hline & $25+$ & $19-24$ & $13-18$ & $7-12$ & $1-6$ & & $1-6$ & $7-12$ & $13-18$ & $19-24$ & $25+$ & \\
\hline Earlier maturers & & & & 2 & 10 & 2 & 14 & 21 & 19 & 23 & 37 & 128 \\
\hline Later maturers & 9 & 9 & 18 & 18 & 26 & 7 & 17 & 23 & 8 & 10 & 1 & 146 \\
\hline The total number of observed cases & 9 & 9 & 18 & 20 & 36 & 9 & 31 & 44 & 27 & 33 & 38 & 274 \\
\hline
\end{tabular}


confirms that more participation was obtained from latermaturing girls than earlier-maturing girls in the pre-menarcheal data, and vice versa in the post-menarche data, both groups covered the transition from pre- to post-menarche. However, caution should be exercised in interpreting the results, as the number of observations for earlier-maturing girls in the pre-menarcheal period, in particular, was small.

\section{Covariates}

\section{Frequency of Physical Abuse}

Multiple subtypes of maltreatment often co-occur in a single case of childhood abuse (Manly et al. 2001). In the current sample, girls with CSA had been physically abused more frequently $(M=1.21)$ than the girls without any CSA $(M=0.58), F(1,91)=5.03, p<.05$. Therefore, we included the number of physical abuse incidents as a covariate, derived from the same MCS coding system as described above for CSA. There were no statistical differences in the frequency of other subtypes of maltreatment between girls with CSA and those without; thus, they were not included in the analyses.

\section{Chronological Age}

We statistically controlled for age at $\mathrm{T} 1$ in the analytical models.

\section{Intervention Condition}

Although intervention effects were not a focus of the present study and were not hypothesized to relate to the study variables, we took the intervention condition into account as a clustering variable in our analyses.

\section{The Analytical Strategy}

\section{Study Design}

The primary mission of longitudinal studies is to examine intra-individual change over time and inter-individual differences in the changes (Baltes and Nesselroade 1979). The present study is no exception; the aim of this study was to examine trajectories of anxiety during the menarcheal transition. However, achieving this goal necessitated special care in the analytical strategy. Two analytical strategies were adopted: the repeated assessment of menarcheal information and the use of gynecological age as a time unit of analysis. These two strategies allowed us to take a development approach and examine changes in anxiety symptoms in the pre-, peri-, and post-menarcheal phases.
In order to investigate the ebbs and flows of anxiety during the transition, it is important that the assessment schedule captures anxiety before, during, and after girls' menarche. This is a complicated task because of the wide variation in the age at which girls reached menarche. The majority of menarche research tends to use retrospective report of menarche, which inevitably limits the assessment of "current" anxiety to the post-menarche period. As mentioned previously, although some girls in the analytical sample had reached the post-menarcheal status by the time the study began, the majority $(73 \%)$ of the girls in our sample were premenarcheal at the outset of the study. We repeatedly asked detailed questions about menarche over several data assessments in an effort to capture their transition from pre- to post-menarcheal status.

The second analytical approach we adopted to capture girls at the menarcheal transition is the use of gynecological age, instead of chronological age, as a time unit of analysis. Researchers interested in intra-individual changes over time typically analyze data by examining how individuals of roughly the same age change over multiple waves of data collection. In such cases, the unit of analysis (or $\mathrm{X}$-axis on the graph that describes an age trend) is chronological age. While chronological age is the fundamental unit to describe development, the meaning attached to age can sometimes be opaque (Rutter 1989). The questions addressed in the present study represent one of those cases; simply describing age-dependent trends is not a sufficient way to illustrate how individuals change before, during, and after an event (e. g. menarche). Thus, we use gynecological age (i.e., the time interval between menarche and the assessment of anxiety for each individual) as a time scale. Figure 1 illustrates two hypothetical cases in which anxiety symptoms of two girls of the same age are assessed at age 11, 12, and 13 for T1, T2, and T3, respectively. For Subject A (an earlier maturer who had menarche at age 11), the T1 assessment reflects data collected at the year of menarche. The T2 and T3 assessments were 1 and 2 years after she experienced her first menstrual cycle, respectively. For Subject B, who had menarche at age 12, the T1 assessment was conducted 1 year before she had menarche; the T2 and T3 assessments represent the year of menarche and 1 year after her first menstrual cycle, respectively. Essentially, this strategy is analogous to centering the time unit at the age of menarche (i.e., peri-menarche).

Although all participants were interviewed at the same calendar time (i.e., at baseline, 12 months, and 24 months), the timing of their interviews relative to age of menarche differed due to the natural variability in the age of menarche. Thus, the timing of assessment in the present study was variant. In other words, the number of assessments is equal for everyone, but the anxiety data were not collected at the same time (in relation to menarche) for all 
Fig. 1 Illustration of the study design with examples (gynecological age, time of anxiety assessments, and menarcheal timing)

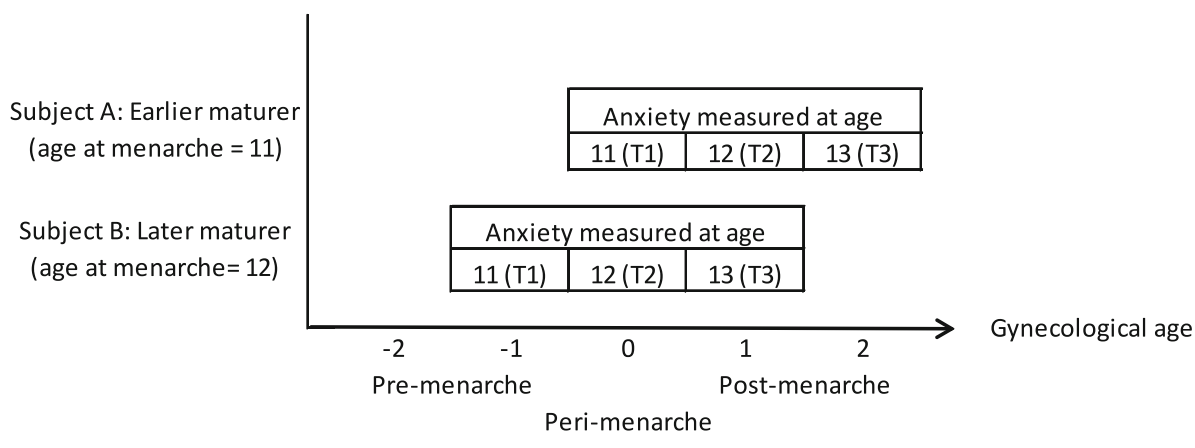

examination of trajectories in anxiety at three time segments: changes in anxiety during the pre-menarche period (Slope A), anxiety at the peri-menarche transition (the intercept), and changes in anxiety during the post-menarche period (Slope B). We conducted two-slope growth curve analysis using PROC MIXED in SAS9.2 (SAS Institute Inc. 2010). In the estimation process, we allowed the variance between the intercept and two slopes to covary.

The analyses were organized as follows. First, descriptive statistics were reported. Second, a series of two-slope growth curve analyses were performed to test whether menarcheal timing and CSA were associated with the trajectories of anxiety during the pre-, peri-, and post-menarcheal phases.

\section{Results}

\section{Descriptive Analyses}

Figure 2 illustrates the mean changes in anxiety symptoms over the course from the pre- to post-menarcheal phases by the status of menarcheal timing. The trend in the mean values suggests several interesting patterns: (a) during the pre-menarche phase, earlier-maturing girls appeared to show higher levels of anxiety than later-maturing girls; and (b) anxiety declined in earlier-maturing girls during the post-menarcheal phase. In general, these descriptive findings suggest that anxiety seemed to change during this developmental transition and that the means and slopes of anxiety may differ by the status of menarcheal timing. These observations were tested formally in the analyses described below.

\section{Primary Analyses}

To investigate changes in anxiety symptoms during the transition from pre- to post-menarche, we performed a series of two-slope growth curve models. The results are presented in Table 3. Estimated coefficients are reported in unstandardized form, indicating the degree of increases (or 
Fig. 2 Means of anxiety symptoms during the transition from pre- to post-menarche
TIMING AND TRANSITION OF MENARCHE

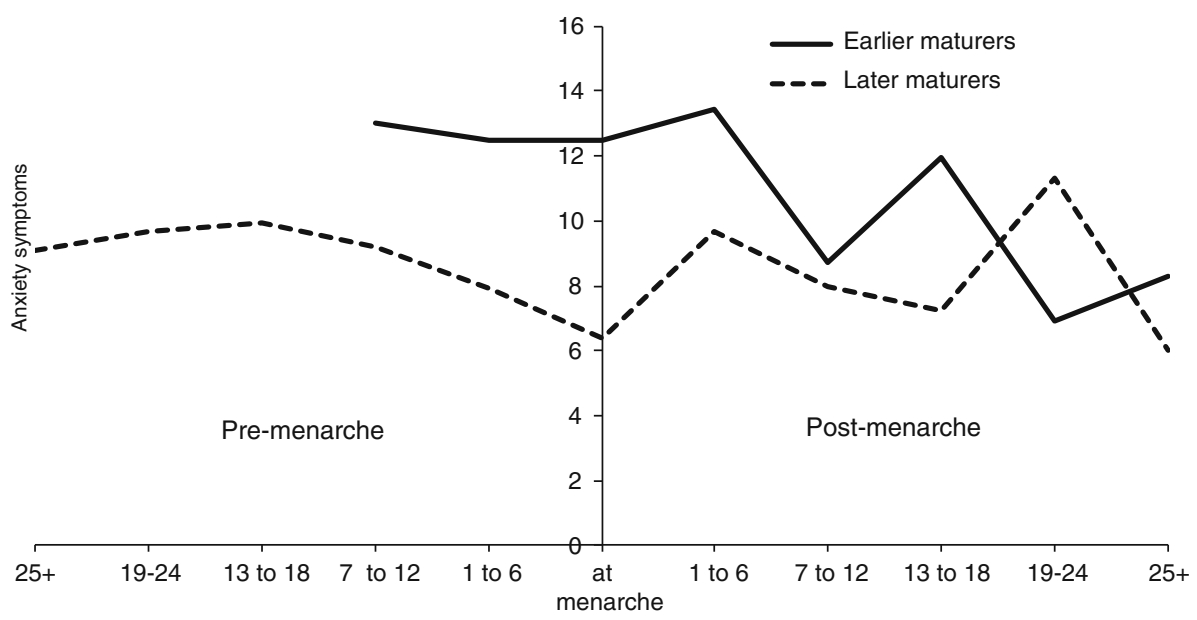

Gynecological age (in months) decreases) in the dependent variable (i.e., anxiety symptoms) with a unit increase in their respective predictors. To facilitate the interpretation of the intercept, age at the T1 assessment was centered at 11.5 years (the mean age at T1). Model 1 was designed to answer the following questions: How do the levels of anxiety symptoms change during the transition from pre- to post-menarche? Are girls who experienced menarche earlier than their peers and those who have a history of CSA more anxious during this developmental transition? The results indicated that, on average, anxiety symptoms decreased during both pre- and post-menarcheal phases $(b=-0.81, p<.05, b=-0.61$, $p<.01$, respectively). The main effect of early menarche was statistically significant $(b=2.23, p<.05)$, indicating that girls who experienced menarche earlier than their peers were at an increased risk of anxiety throughout the transition. Girls with a history of CSA also reported higher levels of anxiety symptoms than girls without any history of CSA over the course of the transition $(b=3.28$, $p<.01)$.

The significant main effect of earlier menarche was expanded in Model 2 by estimating its cross-level interaction effect with the slopes of the pre- (Slope A) and postmenarcheal (Slope B) phases. Because of the inclusion of these cross-level interactions (i.e., Slope $\mathrm{A} \times$ Earlier Menarche; Slope $\mathrm{B} \times$ Earlier Menarche), which were missing in Model 1, the significant main effect of earlier menarche in Model 2 specifically tests whether earliermaturing girls experienced more anxiety at the peri-menarcheal phases than girls who matured later. The main effect of earlier menarche was indeed significant $(b=4.47$, $p<.01$ ), suggesting that the peri-menarcheal phase was more anxiety-laden (a 4.47-unit increase in anxiety symptoms) for earlier-maturing girls than for later-maturing girls. Furthermore, we found that one of the interaction terms, i.e.,
Slope $\mathrm{B} \times$ Earlier menarche, was statistically significant ( $b=-1.10, p<.05$; Fig. 3). As shown in Fig. 3, earliermaturing girls showed a steeper decline in anxiety during the post-menarcheal phase compared to later-maturing girls.

Finally, Model 3 was designed to follow up on the effect of CSA observed in Model 1. Specifically, it examined how CSA was associated with the changes in anxiety during the transition from pre- to post-menarche. The main effect of CSA was significant $(b=4.44, p<.01)$, indicating that compared to girls who did not experience CSA, girls who had experienced CSA showed more anxiety symptoms during the peri-menarcheal phase. However, no statistically meaningful cross-level interaction (i.e., Slope $\mathrm{A} \times \mathrm{CSA}$, Slope B $\times$ CSA) was detected.

\section{Discussion}

Menarche is a developmentally salient demarcation in a female's life course that may precipitate a sudden change in awareness of maturation. In the past two decades, examination of how girls react to this abrupt change in their body has been dormant in the puberty literature. This study suggests a revived need for research attention in this domain by offering a new perspective on how the experiences of this transition may vary according to the timing of its occurrence and contextual circumstances. Capitalizing on prospective longitudinal data that followed pubescent girls with histories of childhood maltreatment, this study addressed several questions about developmental changes in anxiety throughout the course of the menarcheal transition. To our knowledge, the present study is among the first to consider trajectories of anxiety and the transitional and timing aspects of menarche simultaneously, while also taking individual developmental history into account. 
Fig. 3 Estimated trajectories of anxiety symptoms during the transition from pre- to postmenarche

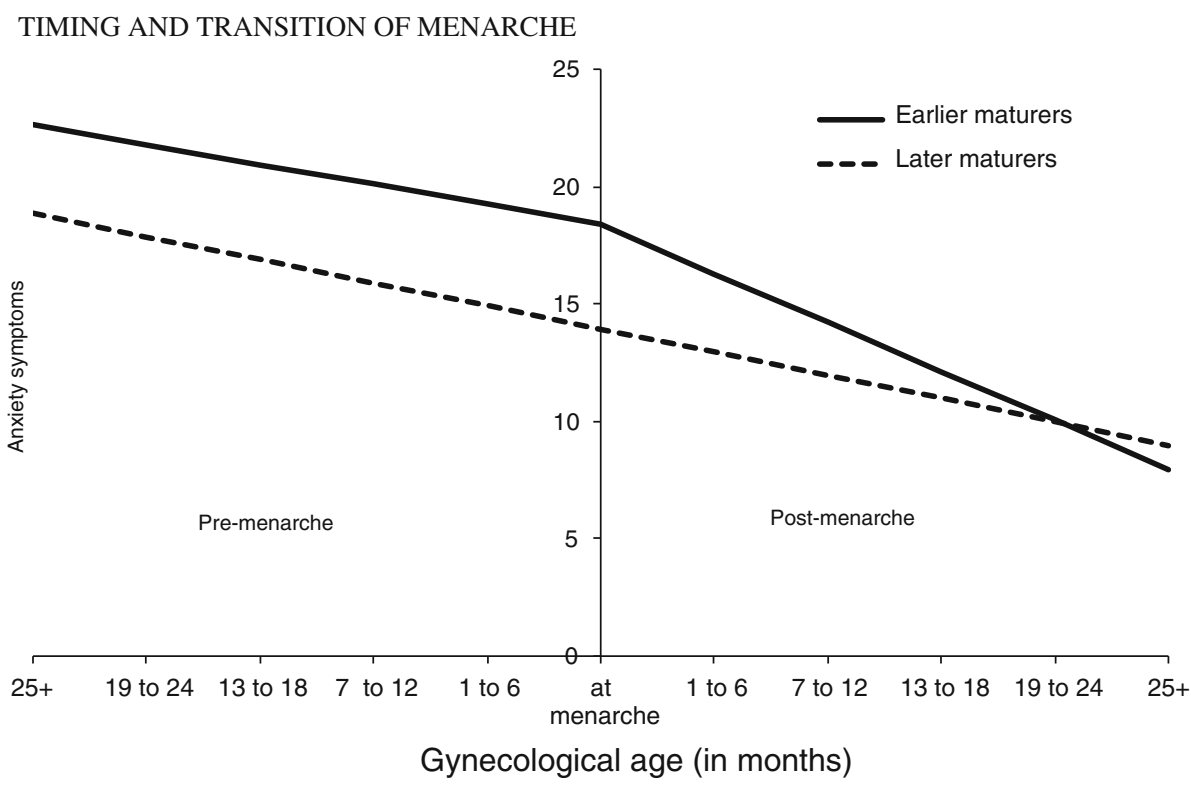

Table 3 The effects of menarcheal timing and childhood sexual abuse during the transition from pre- to post-menarche

\begin{tabular}{|c|c|c|c|c|c|c|}
\hline & \multicolumn{2}{|l|}{ Model 1} & \multicolumn{2}{|l|}{ Model 2} & \multicolumn{2}{|l|}{ Model 3} \\
\hline & $b$ & SE & $b$ & SE & $b$ & SE \\
\hline \multicolumn{7}{|l|}{ Fixed effects } \\
\hline Intercept & $12.95 * * *$ & 1.09 & $13.93 * * *$ & 1.25 & $13.23 * * *$ & 1.13 \\
\hline Age & $3.34 * * *$ & 1.07 & $3.25 * * *$ & 1.07 & $3.30 * * *$ & 1.07 \\
\hline Physical abuse & $-0.94 * *$ & 0.37 & $-0.89 * *$ & 0.38 & $-0.94 * *$ & 0.38 \\
\hline Slope A (pre-menarche) & $-0.81 * *$ & 0.33 & -0.99 & 1.26 & $-0.83 *$ & 0.45 \\
\hline Slope B (post-menarche) & $-0.61 * * *$ & 0.23 & $-0.99 * * *$ & 0.31 & $-0.77 * * *$ & 0.26 \\
\hline Earlier menarche & $2.23 * *$ & 1.08 & $4.47 * * *$ & 1.54 & $2.16^{* *}$ & 1.09 \\
\hline Sexual abuse & $3.28 * * *$ & 1.10 & $3.08 * * *$ & 1.10 & $4.44 * * *$ & 1.63 \\
\hline Slope A $\times$ Earlier menarche & & & 0.14 & 1.32 & - & - \\
\hline Slope B $\times$ Earlier menarche & & & $-1.10 * *$ & 0.48 & - & - \\
\hline Slope A $\times$ Sexual abuse & & & - & - & 0.09 & 0.67 \\
\hline Slope B $\times$ Sexual abuse & & & - & - & -0.64 & 0.50 \\
\hline \multicolumn{7}{|l|}{ Random effects } \\
\hline Variance intercept & $23.71 * * *$ & & $23.13 * * *$ & & $23.87 * * *$ & \\
\hline Variance slope A & $1.97 *$ & & $2.18^{*}$ & & $1.99 *$ & \\
\hline Variance slope B & $1.26 * *$ & & $1.17 * *$ & & $1.24 * *$ & \\
\hline Residual & $12.94 * * *$ & & $12.46 * * *$ & & $12.76 * * *$ & \\
\hline
\end{tabular}

$* p<.10 ; * * p<.05 ; * * * p<.01$

Results indicate that, overall, girl's anxiety symptoms declined during the menarcheal transition. This finding recapitulates an earlier observation that anticipation of menarche is more difficult and anxiety-provoking than the actual experience (Brooks-Gunn and Ruble 1982). In addition, our anxiety findings are consistent with past work in that menarche is not a crisis event that necessarily or permanently disrupts the emotional well-being of girls at this age (Brooks-Gunn and Ruble 1982; Ruble and
Brooks-Gunn 1982). Earlier-maturing girls were more anxious before and at menarche than were later-maturing girls. This finding echoes previous findings that menarche is most challenging for those who feel unprepared for it (Ruble and Brooks-Gunn 1982), and is also congruent with the broad literature on the risk associated with early pubertal timing and subsequent adjustment (Ge and Natsuaki 2009; Mendle et al. 2007). In the absence of experiences and precedents in same-aged peers, the anxiety of earlier-maturing girls 
associated with a feeling of unpreparedness may be accentuated. This notion is consistent with Neugarten's (1976) broader theoretical framework, in that in a society in which life events are ordered in an age-graded timetable, the adaptation and coping skills of individuals are tested when normative life events occur outside of the given timeframe. Importantly, the effect of menarcheal timing remained significant even after controlling for the effects of CSA, which demonstrates a unique effect of early menarche on trajectories of anxiety.

Although earlier-maturing girls may have been more anxious than later-maturing girls, the levels of their anxiety did not remain consistently high over the course of the transitional period. In fact, the pace of the post-menarcheal decline in anxiety was faster for earlier-maturing girls than for their later-maturing counterparts. The mechanisms of this rapid decline provide a clear vein of inquiry for future research. As discussed above, departures from socially normative timing and deviance from the expected schedule of life events are believed to be stressful (Neugarten 1976). However, importantly, the reverse may also be true; specifically, the regaining of a normative status may lead to decreases in distress. Menarche is a universal biological phenomenon that all healthy females will eventually experience. While earlier-maturing girls may deviate from the norm at young ages, their post-menarcheal status eventually becomes the norm, as increasing numbers of peers reach menarche and enter the post-menarcheal phase. The downward changes in the anxiety levels of earliermaturing girls may correspond to their gradual gain of a normative status as the maturational level of their peers catch up with them. Further investigation is encouraged to examine how developmental changes from the "deviant" to the "normative" status in pubertal maturation affect the emotional adjustment of adolescents. The findings on menarcheal transition (i.e., girls' levels of anxiety were higher during pre- and peri-menarcheal phases than the post-menarcheal phase) and timing (i.e., compared to later maturers, earlier maturers were more anxious during the transition, but their anxiety declined faster in the postmenarcheal phase) support the idea that both the timing and transition of menarche need to be considered when understanding the emotional experiences of girls in this critical developmental milestone.

Finally, the third question that guided this study focused on the contribution of individual developmental circumstances to emotional adjustment during the menarcheal transition. We found that the experience of CSA imposed a significant risk for the development of anxiety symptoms throughout the course of the menarcheal transition. Girls with a history of CSA exhibited more severe anxiety symptoms than those without such histories, even in a sample where all participants were residing in foster care and had previous experiences of childhood maltreatment. Their elevated anxiety symptoms did not seem to dissipate faster, which suggests that their anxiety symptoms were consistently higher than those of girls who did not experience any CSA. It is important to note that all of the girls in our sample were maltreated during childhood and that the effect of CSA was examined in comparison to other types of child maltreatment that were non-sexual in nature (e.g., physical abuse, neglect, emotional abuse). Thus, the current finding implies that CSA has a unique contribution in the development of anxiety during the menarcheal transition that non-sexual childhood maltreatment does not have. One possible explanation of this finding is that menarcheal transition is a sexual event that reawakens unpleasant childhood memories and traumas that developed at the time of their abusive experience(s) (Noll et al. 2003).

Our study has several unique strengths. First, we used the official records of childhood maltreatment. Although the official records likely underestimate the cases of childhood maltreatment (because they do not capture the cases that have been unreported), they offer an advantage over self reports which are more common in the CSA literature; the information about maltreatment is more free of memory and perceptual biases of the victims. Second, most of the girls were premenarcheal at the outset of the study and menarche and anxiety were repeatedly assessed over the course of the study period. This strategy permitted us to capture the changes in menarcheal status as well as to obtain the data on menarcheal age as recent as possible. Finally, finding an effect of pubertal timing in a sample of girls who were raised in very stressful family environment suggests the robustness of the effects of menarcheal timing on girls' anxiety.

\section{Limitations}

In considering the present results, several caveats should be noted. First, as with many other studies, results may not be generalized beyond the population represented by the sample, which was girls with a history of childhood maltreatment and placement in foster care. In particular, the absence of a non-maltreated comparison group constrains the generalizability of the findings. However, the nature of the sample enabled us to uniquely examine developmental questions regarding the contribution of CSA to anxiety trajectories and age of menarche. Second, as in many studies recruiting a high-risk sample, our sample size was smaller than ideal, limiting statistical power. In particular, pre-menarcheal anxiety data for earlier-maturing girls were under-represented. Thus, caution should be exercised in interpreting the results regarding earlier-maturing girls' 
anxiety in the pre-menarcheal period. Additionally, only one-third of the participants $(n=31)$ were the victims of maltreatment without sexual abuse. Replication with samples similar in size or larger than the current study is warranted and would increase the interpretive conclusions that can be drawn. We also recognize that the observed effects are small in magnitude. However, it should be emphasized that because of complexity in human behaviors and emotions, the effect size is necessarily small in outcomes with multiple determinants (Ahadi and Diener 1989). Finally, due to the small sample size, we classified menarcheal timing in the form of a dichotomous scale (earlier- or later-maturing girls), which may have decreased the sensitivity in detecting delicate relationships between age at menarche and anxiety symptoms. Nonetheless, the validity of the classification, particularly for the earliermaturing group, was strengthened by the fact that the average age at menarche for girls in the earlier-maturing group was much earlier than the national average (12.4 years old; McDowell et al. 2007). This is important because the reference group to which girls compare their bodies in everyday life is peers from a general population, not peers within a population of victims of childhood maltreatment.

\section{Conclusion}

The current investigation accentuates the importance of the intricate developmental relationships between menarcheal timing, personal maltreatment history, and anxiety during the transition from pre- to post-menarche. Our findings demonstrate that the menarcheal transition is particularly difficult and anxiety-provoking for girls who have undertaken this transition early and for those with a documented history of CSA. These findings deepen the understanding of developmental mechanisms by documenting that changes and variation in anxiety during the menarcheal transition involve not only the timing of menarche but also girls' pre-pubertal family history (i.e., CSA experiences). If replicated, the current findings suggest several potential intervention avenues. First, the decline in anxiety for all participants (regardless of CSA experiences) during the peri- and post-menarcheal periods suggests that interventions aimed at reducing anxiety might have the most benefit when implemented at the earliest stages of pubertal development, prior to onset of menarche. Second, such interventions would have the most benefit for girls who are showing signs of pubertal development earlier than their peers. Third, because CSA experiences are also associated with increased anxiety trajectories, anxiety-prevention focused interventions for this subgroup of girls might be especially beneficial in preventing the elevation in anxiety across adolescence that is often seen in CSA-exposed girls. Additional investigations into the long-term anxiety trajectories as a function of CSA experiences and pubertal timing are needed to provide further insights about opportunities for and potential benefits of anxiety-focused interventions for this vulnerable population.

Acknowledgments Support for this research was provided by R01 MH054257, NIMH, U.S. PHS and R21 DA027091 U.S. PHS. Additional support for the writing of this article was provided by R01 DA024672 and P30 DA023920, NIDA, U.S. PHS. We would like to thank the foster families who participated in this study and the Oregon Department of Human Services for their generous assistance with the project. We are also grateful to Patricia Chamberlain and Dana Smith for their scientific contributions to the overall study, to Gordon Harold for his helpful comments on an earlier version of the paper, and to Heather Wilber, Courtenay Paulic, and Michelle Baumann for their assistance with technical aspects of this project.

Open Access This article is distributed under the terms of the Creative Commons Attribution Noncommercial License which permits any noncommercial use, distribution, and reproduction in any medium, provided the original author(s) and source are credited.

\section{References}

Ahadi, S., \& Diener, E. (1989). Multiple determinants and effect size. Journal of Personality and Social Psychology, 56, 398-406.

Baltes, P. B., \& Nesselroade, J. R. (1979). History and rationale of longitudinal research. New York: Academic Press.

Beitchman, J. H., Zucker, K. J., Hood, J. E., DaCosta, G. A., Akman, D., \& Cassavia, E. (1992). A review of the long-term effects of child sexual abuse. Child Abuse and Neglect, 16, 101-118.

Bell, R. Q. (1953). Convergence: An accelerated longitudinal approach. Child Development, 24(2), 145-152.

Belsky, J., Steinberg, L., \& Draper, P. (1991). Childhood experience, interpersonal development, and reproductive strategy: An evolutionary theory of socialization. Child Development, 62, $647-670$.

Bergevin, T. A., Bukowski, W. M., \& Karavasilis, L. (2003). Child sexual abuse and pubertal timing: Implications for long-term psychosocial adjustment. In C. Hayward (Ed.), Gender differences at puberty (pp. 187-216). Cambridge, England: Cambridge University Press.

Brooks-Gunn, J., \& Petersen, A. C. (Eds.). (1983). Girls at puberty: Biological and psychosocial perspectives. New York: Plenum Press.

Brooks-Gunn, J., \& Ruble, D. N. (1982). The development of menstrual-related beliefs about behaviors during early adolescence. Child Development, 53, 1567-1577.

Caspi, A., \& Moffitt, T. E. (1991). Individual differences are accentuated during periods of social change: The sample case of girls at puberty. Journal of Personality and Social Psychology, 61, 157-168.

Caspi, A., \& Moffitt, T. E. (1993). When do individual differences matter? A paradoxical theory of personality coherence. Psychological Inquiry, 4, 247-271.

Chamberlain, P., Leve, L. D., \& Smith, D. K. (2006). Preventing behavior problems and health-risking behaviors in girls in foster care. International Journal of Behavioral and Consultation Therapy, 4, 518-530. 
Chumlea, W. C., Schubert, C. M., Roche, A. F., Kulin, H. E., Lee, P. A., Himes, J. H., et al. (2003). Age at menarche and racial comparisons in US girls. Pediatrics, 111, 110-113.

Conley, C. S., \& Rudolph, K. D. (2009). The emerging sex differences in adolescent depresion: Interacting contributions of puberty and peer stress. Development and Psychopathology, $21,593-620$.

Dorn, L. D., Negriff, S., Huang, B., Pabst, S., Hillman, J., Braverman, P., et al. (2009). Menstrual symptoms in adolescent girls: Association with smoking, depressive symptoms, and anxiety. Adolescent Health, 44, 237-243.

Duncan, S. C., Duncan, T. E., \& Hops, H. (1996). Analysis of longitudinal data within accelerated longitudinal designs. Psychological Methods, 1(3), 236-248.

Ellis, B. J. (2004). Timing of pubertal maturation in girls: An integrated life history approach. Psychological Bulletin, 130, 920-958.

Finkel, D., Reynolds, C. A., McArdle, J. J., Gatz, M., \& Pedersen, N. L. (2003). Latent growth curve analyses of accelerating decline in cognitive abilities in late adulthood. Developmental Psychology, 39, 535-550.

Ge, X., \& Natsuaki, M. N. (2009). In search of explanations for early pubertal timing effects of developmental psychopathology. Current Directions in Psychological Science, 18, 327-331.

Graber, J. A. (2003). Puberty in context. In C. Hayward (Ed.), Gender differences at puberty (pp. 307-325). New York: Cambridge University Press.

Graber, J. A., Seeley, J. R., Brooks-Gunn, J., \& Lewinsohn, P. M. (2004). Is pubertal timing associated with psychopathology in young adulthood? Journal of the American Academy of Child and Adolescent Psychiatry, 43, 718-726.

Hale, W. W., Raaijmakers, Q., Muris, P., van Hoof, M., \& Meeus, W. (2008). Developemtnal trajectories of adolescent anxiety disorder symptoms: A 5-year prospective community study. Journal of American Academy of Child and Adolescent Psychiatry, 47, $556-564$.

Hayward, C., Killen, J. D., Hammer, L. D., Litt, I. F., Wilson, D. M., Simmonds, B., et al. (1992). Pubertal stage and panic attack history in 6th -grade and 7th grade girls. American Journal of Psychiatry, 149, 1239-1243.

Kaltiala-Heino, R., Marttunen, M., Rantanen, P., \& Rimpela, M. (2003). Early puberty is associated with mental health problems in middle adolescence. Social Science and Medicine, 57, $1055-1064$.

Manly, J. T., Kim, J. E., Rogosch, F. A., \& Cicchetti, D. (2001). Dimensions of child maltreatment and children's adjustment: Contributions of developmental timing and subtype. Development and Psychopathology, 13, 759-782.

McArdle, J. J., Ferrer-Caja, E., Hamagami, F., \& Woodcock, R. W. (2002). Comparative longitudinal structural analyses of the growth and decline of multiple intellectual abilities over the life span. Developmental Psychology, 38, 115-142.

McDowell, M. A., Brody, D. J., \& Hughes, J. P. (2007). Has age at menarche changed? Results from the National Health and Nutrition Examination Survey (NHANES) 1999-2004. Journal of Adolescent Health, 40, 227-231.

Mendle, J., Leve, L. D., Van Ryzin, M., Natsuaki, M. N., \& Ge, X. (2010). Association between early life stress, child maltreatment, and pubertal development. Journal of Research on Adolescence (in press).

Mendle, J., Turkheimer, E., \& Emery, R. E. (2007). Detrimental psychological outcomes associated with early pubertal timing in adolescent girls. Developmental Review, 27, 151-171.

Miyazaki, Y., \& Raudenbush, S. W. (2000). Tests for linkage of multiple cohorts in an accelerated longitudinal design. Psychological Methods, 5(1), 44-63.
Moore, S. M. (1995). Girls' understanding and social constructions of menarche. Journal of Adolescence, 18, 87-104.

Neugarten, B. L. (1976). Adaptation and the life cycle. Counseling Psychologist, 6, 16-20.

Noll, J. G., Trickett, P. K., \& Putnam, F. W. (2003). A prospective investigation of the impact of childhood sexual abuse on the development of sexuality. Journal of Consulting and Clinical Psychology, 71, 575-586.

Overton, W. F. (1998). Developmental psychology: Philosophy, concepts, and methodology. In W. Damon \& R. M. Lerner (Eds.), Handbook of child psychology: Theoretical models of human development (5th ed., Vol. 1, pp. 109-188). New York: Wiley.

Petersen, A. C., Crockett, L. J., Richards, M., \& Boxer, A. (1988). A self-report measure of pubertal status: Reliability, validity, and initial norms. Journal of Youth and Adolescence, 17, 117-133.

Pickles, A., Neale, M., Simonoff, E., Rutter, M., Hewitt, J., Meyer, J., et al. (1994). A simple method for censored age-of-onset data subject to recall bias: Mothers' reports of age of puberty in male twins. Behavioral Genetics, 24, 457-468.

Raudenbush, S. W., \& Bryk, A. S. (2002). Hierarchical linear models: Applications and data analysis methods (2nd ed.). Thousand Oaks, CA: Sage Publication.

Reardon, L. E., Leen-Feldner, E. W., \& Hayward, C. (2009). A critical review of the empirical literature on the relation between anxiety and puberty. Clinical Psychology Review, 29, 1-23.

Rembeck, G. I., Moller, M., \& Gunnarsson, R. K. (2006). Attitudes and feelings towards menstruation and womanhood in girls at menarche. Acta Pediatrica, 95, 707-714.

Reynolds, C. R. (1981). Long-term stability of scores on the Revised Children's Manifest Anxiety Scale. Perceptual and Motor Skills, 53, 702 .

Reynolds, C. R., \& Paget, K. D. (1983). National normative and reliability data for the Revised Children's Manifest Anxiety Scale. School Psychology Review, 12, 324-336.

Reynolds, C. R., \& Richmond, B. O. (1978). What I think and feel: A revise measure of children's manifest anxiety. Journal of Abnormal Child Psychology, 6, 271-280.

Reynolds, C. R., \& Richmond, B. O. (1979). Factor structure and construct validity of "What I Think and Feel": The Revised Children's Manifest Anxiety Scale. Journal of Personality Assessment, 43, 281-283.

Romans, S. E., Martin, M., Gendall, K., \& Herbison, G. P. (2003). Age of menarche: The role of some psychosocial factors. Psychological Medicine, 33, 933-939.

Ruble, D. N., \& Brooks-Gunn, J. (1982). The experience of menarche. Child Development, 53, 1557-1566.

Rudolph, K. D., \& Troop-Gordon, W. (2010). Personal-accentuation and contextual-amplification models of pubertal timing: Predicting youth depression. Development and Psychopathology, 22, 443-451.

Rutter, M. (1989). Age as an ambiguous variable in developmental research: Some epidemiological considerations from developmental psychopathology. International Journal of Behavioral Development, 12, 1-34.

Rutter, M. (1996a). Continuities, transitions and turning points in development. In M. Rutter \& D. F. Hay (Eds.), Development through life: A handbook for clinicians (pp. 1-25). Oxford, UK: Blackwell.

Rutter, M. (1996b). Transitions and turning points in developmental psychopathology: As applied to the age span between childhood and mid-adulthood. International Journal of Behavioral Development, 19, 603-626.

SAS Institute Inc. (2010). SAS OnlineDoc 9.2. Cary, NC: Author.

Schaie, K. W. (1965). General model for the study of developmental problems. Psychological Bulletin, 64, 92-107. 
Silverman, A. B., Reinherz, H. Z., \& Giaconia, R. M. (1996). The long-term sequelae of child and adolescent abuse: A longitudinal community study. Child Abuse and Neglect, 20, 709-723.

Tang, C. S., Yeung, D. Y., \& Lee, A. M. (2003). Psychosocial correlates of emotional responses to menarche among Chinese adolescent girls. Journal of Adolescent Health, 33, 193-201.

Trickett, P. K., \& Putnam, F. W. (1993). Impact of child sexual abuse on females: Toward a developmental, psychobiological integration. Psychological Science, 4, 81-87.

Turner, P. K., Runtz, M. G., \& Galambos, N. L. (1999). Sexual abuse, pubertal timing, and subjective age in adolescent girls: A research note. Journal of Reproductive and Infant Psychology, 17, 111-118.

van Bruggen, L. K., Runtz, M. G., \& Kadlec, H. (2006). Sexual revictimization: The role of sexual self-esteem and dysfunctional sexual behaviors. Child Maltreatment, 11, 131-145.

van Hooff, M. H. A., Voorhosrst, F. J., Kaptein, M. B. H., Hirasing, R. A., Koppenaal, C., \& Schoemaker, J. (2000). Insulin, androgen, and gonadotropin concentrations, body mass index, and waist to hip ratio in the first years after menarche in girls with regular menstrual cycles, irregular menstrual cycles, or oligomenorrhea. Journal of Clinical Endocrinology and Metabolism, 85, 1394-1400.

Wise, L. A., Palmer, J. R., Rothman, E. F., \& Rosenberg, L. (2009). Childhood abuse and early menarche: Findings from the Black Women's Health Study. American Journal of Public Health, 99, $1-7$.

Zehr, J. L., Culbert, K. M., Sisk, C. L., \& Klump, K. L. (2007). An association of early puberty with disordered eating and anxiety in a population of undergraduate women and men. Hormones and Behavior, 52, 427-435.

\section{Author Biographies}

Misaki N. Natsuaki received her Ph.D. in Human Development from University of California, Davis. She is currently an Assistant Professor in Department of Psychology at University of California, Riverside. Her research interests include puberty, developmental psychopathology, and the interplay between biology and environment in the development of anxiety and depression in children and adolescents.

Leslie D. Leve received her Ph.D. in Developmental Psychology from University of Oregon. She is currently a Senior Scientist at the Oregon Social Learning Center and the Center for Research to Practice. Her research interests include child welfare involvement, girls, intervention research, and the interplay between genetic and environmental influences on the development of psychopathology.

Jane Mendle is an Assistant Professor in the Department of Psychology at the University of Oregon. She received her Ph.D. in clinical psychology from the University of Virginia. Her research interests include how early childhood experiences relate to pubertal development, as well as how individual differences at puberty predict adolescent adjustment. 